\title{
Novel Applications of High Altitude Stratospheric Platforms for Traffic Management and Control
}

\author{
Yasser Albagory ${ }^{1}$ \\ Computer Engineering Dept., College of Computers and Information Technology, \\ Taif University, Kingdom of Saudi Arabia ${ }^{1}$
}

Electronics and Communication Dept., Faculty of Electronic Engineering, Menoufia University, Egypt ${ }^{1}$

\begin{abstract}
This paper provides some novel applications of High Altitude Stratospheric Platforms (HASP) for traffic monitoring and management systems. A flying HASP in the stratosphere can provide large area coverage with improved communication performance and is candidate for many applications and scenarios such as flood monitoring, highway traffic monitoring and control, accurate localization of remote accidents, tracking of dangerous wanted vehicles and detection of road faults and floods. The system architecture of HASP for traffic management and control is demonstrated with the proposed applications showing the new provided capabilities and advantages such as localization of vehicles, long endurance operation, controlling of traffic lights over wide area by a single HASP, and efficient traffic data collection.
\end{abstract}

Keywords: High Altitude Stratospheric Platforms, Traffic Management, Flood Detection, Vehicle Tracking

\section{INTRODUCTION}

High Altitude Stratospheric Platforms (HASPs) have attracted great attention for many applications such as wireless and mobile data communications, surveillance, wireless data sensor networks, and many others such as digital video broadcasting [1-4]. HASP has shown in many studies a superior performance when adopted in communication systems compared with satellite and terrestrial communication systems [5] where the high possibility of line-of-sight propagation improves both signal strength and quality of reception. The height of HASP ranges from few kilometres up to 50 kilometres in the stratosphere and can cover several hundreds of kilometres diameter. Surveillance is one of the most important applications of HASP where the low altitude compared with satellite systems offers great resolution and accuracy. It can be used for controlling and managing one of the most growing critical problems in modern life which is traffic congestion. Despite great advances in the automotive industry and the high quality of the roads and the extent of securing its drivers against accidents, it is still the rate of accidents at high values, especially when driving on highways where it is believed that along the way, especially in remote areas, censorship police patrols or surveillance cameras are absent which contributes to the high rate of accidents in these areas as a result of exceeding speed limits on these roads. The aerial platform wireless sensor network provides better reliability and security, where the main sink of traffic data is flying far from eavesdropping or destruction and is capable of covering large areas and can link areas that can't be reached by the conventional methods. Therefore, this paper focuses on the possible applications of HASPs for traffic monitoring and management where it is thoroughly explained and demonstrated. In section II, the system architecture is demonstrated and in section III, the application of controlling traffic lights is explained. Section IV provides a technique to track required dangerous vehicles based on HASP while section V proposes a technique for accidents location detection. Section VI provides a solution to the road faults and flood detection. Finally, section VI concludes the paper.

\section{HASP SYSTEM ARCHITECTURE FOR TRAFFIC MANAGEMENT}

The HASP system can be used in many scenarios or applications as shown in Fig. (1) where the AP can be a sink station that collects or gathers data from traffic sensors located on the roadsides and may have its own sensors such as a high-resolution camera that can monitor wide areas and has the focusing, zooming, and pan/tilt capabilities. Also it can be operated by the traffic control centre on ground to extract the required information. In this section, a variety of possible applications can be realized in the proposed HASP traffic monitoring system such as:

- Controlling traffic lights duration according to the traffic density.

- Tracking of dangerous or wanted vehicles. 
International Journal of Advanced Research in Computer and Communication Engineering

Vol. 9, Issue 1, January 2020

- Detection of accidents and its locations.

- Detection of road faults and informing drivers about the other possible roads.

These applications are supported by many additional capabilities in the HASP traffic system compared to the current conventional systems such as:

- Wide area viewing and monitoring.

- Simultaneous control of several traffic lights or signals from aerial imaging.

- Reduced hardware and ground installations.

- Long endurance operation.

- No need for using helicopters or (Unmanned-Aerial Vehicles) UAVs for traffic surveillance applications.

- Continuous aerial monitoring and efficient data gathering from traffic sensors installed on the roadsides due to the improved communications link.

- The capability of monitoring areas that cannot be reached by conventional techniques.

- The gathered information from multiple HASPs can be processed to extract the required information efficiently.

In the following subsections, some possible applications are explored and demonstrated based on the proposed HASP traffic monitoring system.

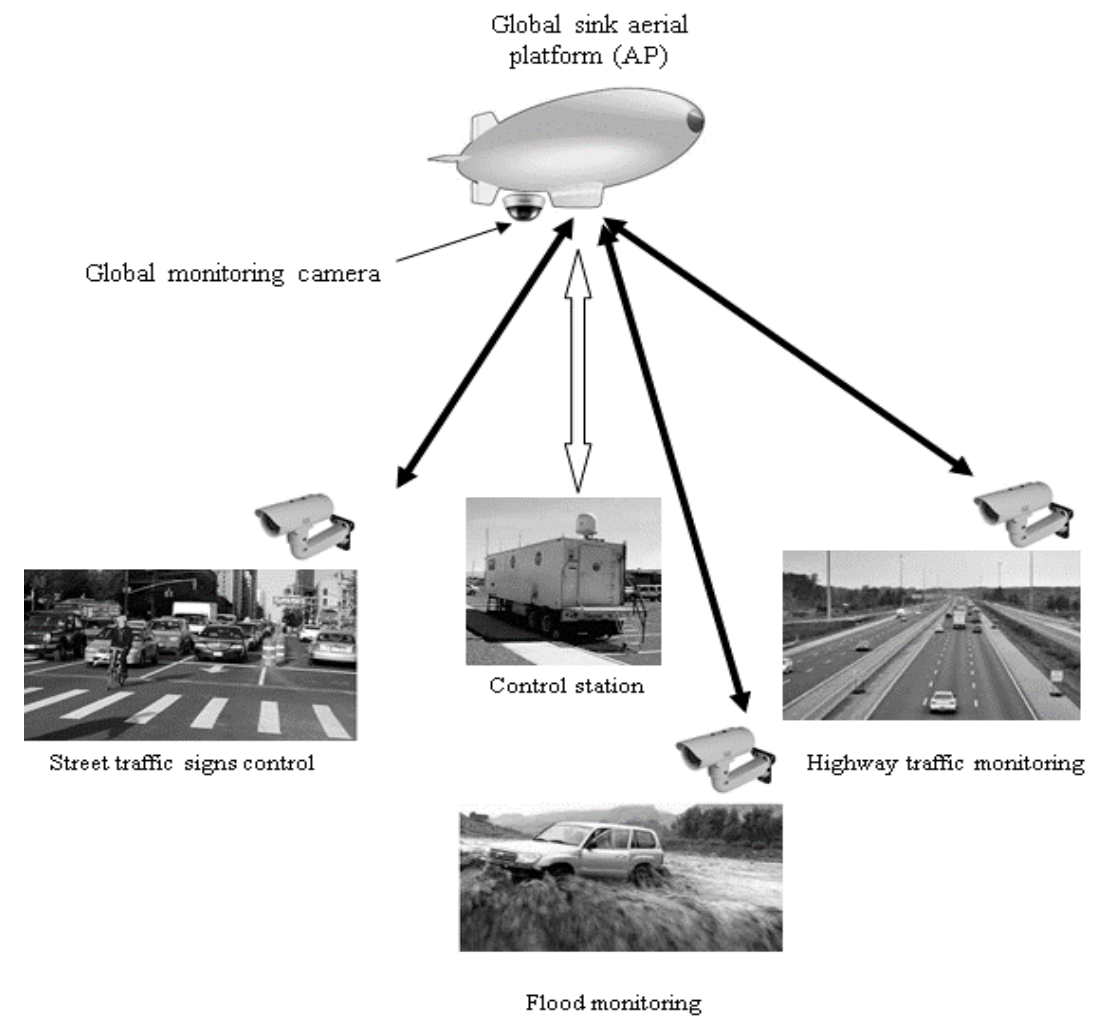

Fig. (1): Traffic monitoring scenarios using HASP

\section{CONTROLLING TRAFFIC LIGHTS (SIGNALS) DURATION ACCORDING TO THE TRAFFIC DENSITY}

Traffic congestion causes many problems such as loss of time, missed opportunities, and frustration [6-8]. One solution to this problem is to adapt the time duration of the traffic signals at the intersections where it can be done manually or automatically. The time duration of the green lights can be adjusted when there is a variable traffic density in its corresponding direction to provide mostly uniform traffic and equal length of vehicles queue. This procedure can be performed efficiently over a wider area and for many traffic intersections at a time by processing aerial images captured from an HASP. The process is investigated in Fig. (2) where an on-board HASP global camera with high-resolution capability monitors a traffic area containing a number of traffic intersections provided that these intersections are clearly viewed by the HASP camera, otherwise the system will depend on the received images from cameras installed at the shadowed intersections. The shadowing of intersections can be also minimized by a proper design of the system where the HASP locations are adjusted to clearly view most of the important traffic areas and intersections. 
The normal traffic densities at each traffic-lights intersection is predetermined in the form of a queue of vehicles and is considered the reference traffic image. Then the identified number of vehicles at any time is then determined and if the new queue of vehicles exceeds the reference one by a certain threshold, the traffic green light is extended by suitable time especially when the other directions have less density. Traffic light management using an on-board HASP camera will provide simultaneous control of traffic lights durations at many traffic intersection points where the whole aerial image can be segmented to provide sub-images at the different intersections which are then processed to determine the required timing information. An HASP will provide better view especially at high-altitudes (several kilometres) and simultaneous view of the four directions at the intersections. These captured images can be processed on-board the HASP and in this case, the traffic control signals can be sent directly from the platform to the control circuits of the traffic lights. Another configuration is to use the HASP station as a sink which collects the captured images from cameras that are held at each traffic intersection and forward it to a traffic control ground centre. The system in this case has to process the individual captured images from their corresponding cameras in each direction which requires combining the results to manage the timing of signals or lights. The control signals of the traffic lights are then sent to the HASP which forwards them to the traffic light control circuits at the corresponding intersections. This configuration is known as bent-pipe communication which resembles satellite broadcasting system.

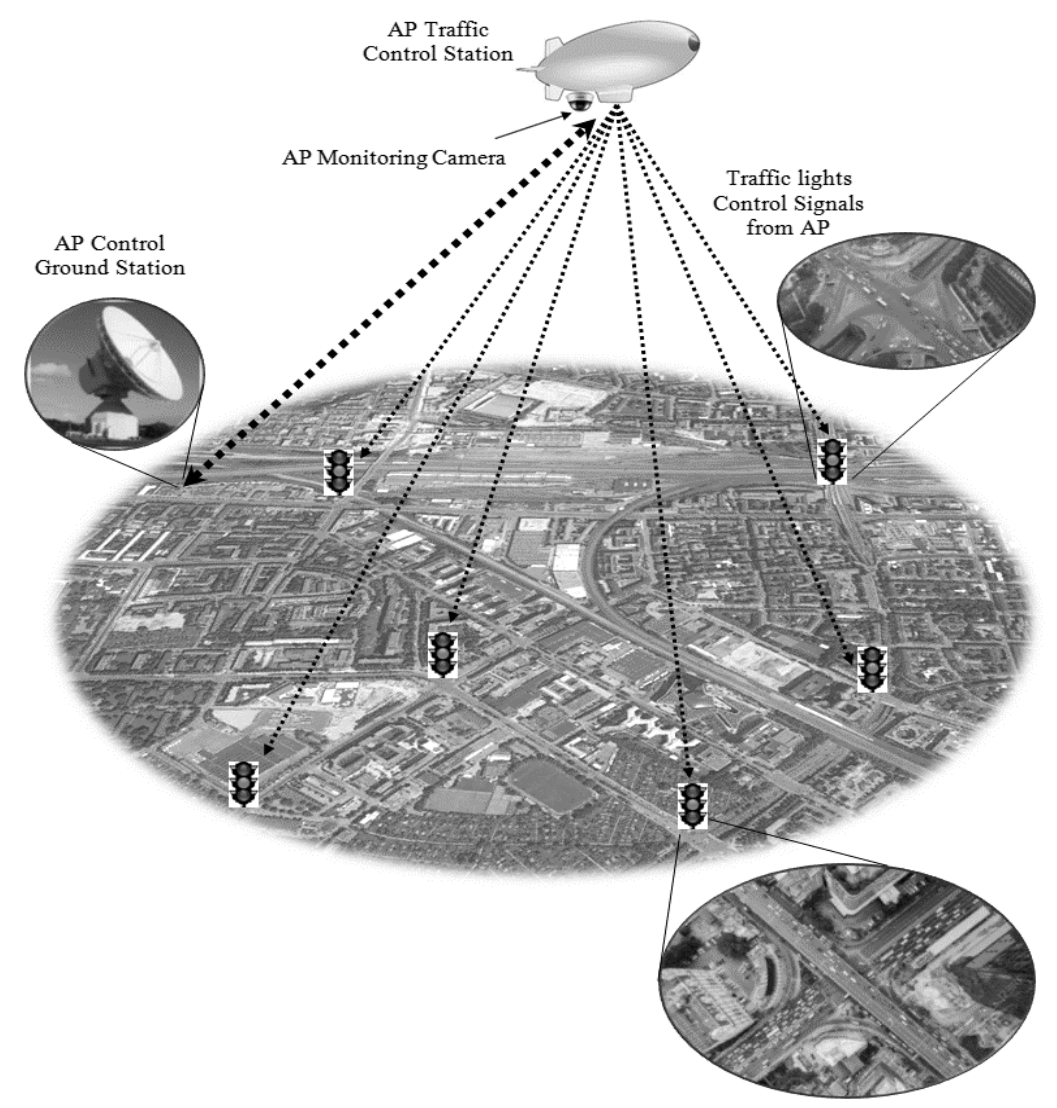

Fig. (2): AP traffic lights control systems.

\section{TRACKING OF DANGEROUS OR WANTED VEHICLES}

One of the most important applications of HASP traffic monitoring system is the capability of tracking wanted vehicles through aerial imaging. The current systems are able to provide this application through tracking by helicopters or UAVs which require continuous flying and is limited by available amount of fuel and the human bearing of the pilot. In the proposed AP traffic monitoring system as shown previously in Fig. (1), the aerial stations (HASPs) can provide wide-area aerial images that can be processed to track the wanted vehicles. The dangerous vehicles can be recognized by its driving behaviour such as very high driving velocities or driving in an opposite direction. It can be recognized by police on ground or identified from HASP cameras and then continuously track its location. The location information of these wanted vehicles can be extracted by relating the HASP location to the captured images as shown in Fig. (3), where the pixel matrix of the captured images is mapped to a GPS coordinates matrix taking into consideration the HASP height and its GPS coordinates. The identified wanted vehicles can be located on the captured images and contoured by a rectangle by many processing techniques [9-10]. The central pixel of this rectangle is used to determine the vehicle location as each pixel in the captured image has an equivalent GPS coordinates as shown in Fig. (3). 


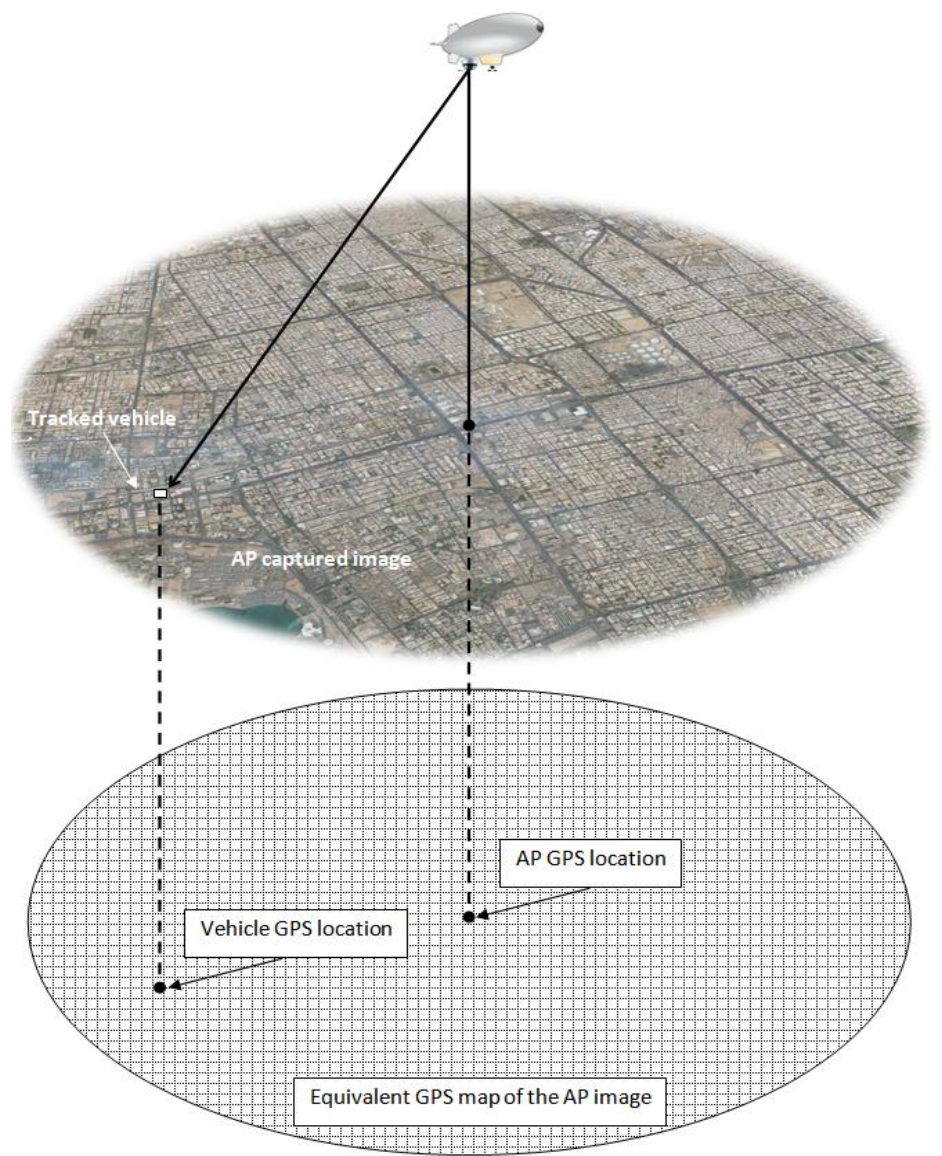

Fig. 3: Vehicles localization from HASP images mapped to GPS coordinates.

\section{DETECTION OF ACCIDENTS AND ITS LOCATION}

An accident in a populated urban area can be reported quickly by other near drivers or pedestrians, but in rarely populated areas or along highways crossing rural areas, it will be very difficult to quickly report about it. One possible solution using the proposed HASP traffic monitoring system is to identify the accidents by the abnormal traffic density over the highways where there will be congestion at the accident location. The detection of an accident should be differentiated from the normal traffic congestion where the accident is characterized by a sudden increase in the traffic density at any time and any location along the road while normal congestions occur during busy hours especially in urban areas, at traffic intersections, and at the entrance or exit of big malls, airports, universities, etc... The detection and identification of accidents using the HASP proposed system will be very effective in rural areas, along highways crossing deserts or roads crossing hilly and mountainous regions and will help police and to identify, track, and rescue unusual grouping of vehicles due to an accident. In the rural areas, it is very difficult to install traffic sensors such as cameras along the highways as there may be no available energy sources and it may be subjected to devastation or damaging, therefore aerial solutions are more suitable, reliable and secure. Many techniques [9] can be applied to identify the vehicles on the roads and contouring them by rectangles. In normal traffic conditions these rectangles are uniformly distributed along the road with almost constant orientation but if there is an accident the rectangles will have different orientations and speeds as shown in Fig. (4). The location of this abnormal vehicle orientation is then identified and forwarded to the rescue or security authorities for handling.

\section{DETECTION OF ROAD FAULTS AND FLOODS}

The aerial images of roads from an HASP can be also processed to identify and detect faults in the roads. The cracks or holes in the roads will force the drivers to change their direction and this action is repeated for most drivers as shown in Fig. (4). With good road condition, the vehicles move almost with uniform orientation along the road lanes. The change in driving direction is detected by comparing the moving vehicle orientation with the lane orientation. If there is any repeated change in direction at a certain point on the road for most of the crossing vehicles, then there will be a high probability of road faults, objects, floods at this point. The main difference between road faults and accidents is that many vehicles may stop for helping the injured people in case of accidents while the drivers in the road faults case will 
Vol. 9, Issue 1, January 2020

drive slowly around these faults and do not stop. Aerial imaging from HASPs can help scan the whole area and capture each subarea with high resolution cameras, then analysing the images to explore the fault points along roads. The reference images should correspond to good road conditions and the subsequent scanned ones are then compared with it. HASP imaging help the identification of road faults quickly due to the broad or coarse imaging nature at high altitudes without the need to install large number of cameras on the ground. The quick identification of road faults helps preventing possible accidents and saves people, time, and money. The roads can be detected using aerial images from UAVs [10]. If a torrent or earthquake happened, then the detected roads will differ from the previously detected ones at normal conditions. The difference between the reference normal road images and the detected ones during floods or earthquakes determines the affected areas and direct the rescue authorities to these affected areas. Using HASP instead of UAV is expected to provide stability in imagery and reliable detection of road faults due to its constant position. On the other hand, vehicle identification in the of flood areas will help in rescue operations of lost drivers where their locations can be determined from the GPS map of the HASP system as shown in Fig. (3).

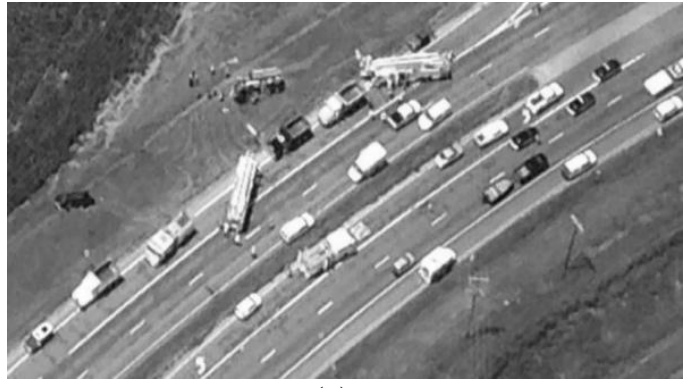

(a)

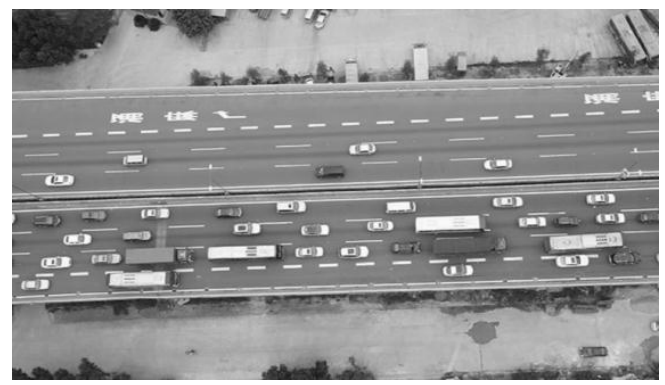

(b)

Fig. (4): Vehicle orientation and distribution along roads in (a) Normal road conditions and (b) Accident conditions.

\section{CONCLUSION}

In this paper, novel applications of High Altitude Stratospheric Platforms (HASP) have been proposed for traffic management and monitoring. The advantages of HASP make it candidate for these proposed techniques where it can be useful in tracking and finding dangerous vehicles, detection on road faults, controlling of traffic lights over large covered area, and in converting aerial images into GPS map for localization purposes. The advantages of the proposed system and applications include long endurance operation, no need for using helicopters or (Unmanned-Aerial Vehicles) UAVs for traffic surveillance applications, continuous aerial monitoring with efficient data harvesting of traffic sensors, the capability of monitoring areas that cannot be reached by conventional techniques. Also, the gathered information from multiple HASPs can be processed to extract the required information efficiently.

\section{REFERENCES}

[1]. A. Mohammed, A. Mehmood, F. Pavlidou and M. Mohorcic "The Role of High-Altitude Platforms (HAPs) in the Wireless Global Connectivity", Proceedings of the IEEE, Vol. 99, No. 11, pp. 1939 - 1953, Nov. 2011.

[2]. Yasser Albagory, Omar Said," Performance enhancement of high-altitude platforms wireless sensor networks using concentric circular arrays", AEU International Journal of Electronics and Communications, Volume 69, Issue 1, January 2015, Pages 382-388.

[3]. Yasser Albagory, Omar Said, Mostafa Nofal, Fahad Alraddady, "An Efficient Traffic Monitoring and Control System using Aerial Platforms and Vertical Arrays" Telecommunication Systems (2018) 69: 131., Springer.

[4]. Yasser Albagory, "An Efficient Technique for Digital Video Broadcasting Using High-Altitude Aerial Platforms and Adaptive Arrays", Progress In Electromagnetics Research M, vol. 61, pp. 97-110, 2017.

[5]. Yang, Z., Mohammed, A. High altitude platforms for wireless sensor network applications. IEEE international symposium on wireless communication systems 2008. ISWCS2008. 2008. P. 613-617.

[6]. A. A. Zaidi, B. Kulcsár and H. Wymeersch, "Back-Pressure Traffic Signal Control with Fixed and Adaptive Routing for Urban Vehicular Networks," in IEEE Transactions on Intelligent Transportation Systems, vol. 17, no. 8, pp. 2134-2143, Aug. 2016

[7]. Mike Smith, "Traffic signal control and route choice, A new assignment and control model which designs signal timings", Transportation Research Part C: Emerging Technologies, Volume 58, Part C, September 2015, Pages 451-473.

[8]. Jens Leitloff, Dominik Rosenbaum, Franz Kurz, Oliver Meynberg and Peter Reinartz, "An Operational System for Estimating Road Traffic Information from Aerial Images", Remote Sensing, vol. 11, pp. 11315-11341, 2014, doi:10.3390/rs61111315.

[9]. Sebastien Razakarivony and Frederic Jurie, "Vehicle detection in aerial Imagery: A small target detection benchmark", Journal of Visual Communication and Image Representation, vol. 34, issue C, pp. 187-203, January 2016.

[10]. Hailing Zhou, Hui Kong, Douglas Creighton and Saeid Nahavandi, "Effecient Road Detection and Tracking for Unmanned Aerial Vehicle", IEEE Transactions on Intelligent Transportation Systems, vol. 16, no. 1, pp. 297-309, February 2015. 